\title{
Experimental study of the spatial evolution of the spectrum of a Raman FEL
}

\author{
P. Zambon ${ }^{\mathrm{a}, *}$, W.J. Witteman ${ }^{\text {a }}$, P.J.M. van der Slot ${ }^{\mathrm{b}}$ \\ a Department of Applied Physics, Unicersity of Twente, P.O. Box 217, 7510 AE Enschede, The Netherlands \\ ${ }^{\mathrm{h}}$ Nederlands Centrum voor Laser Research, P.O. Box 2662, 7500 CR Enschede, The Netherlands
}

\begin{abstract}
For a specific set of parameters, corresponding to an operating point near magneto-resonance, the evolution of the spectrum generated by a Raman FEL was investigated. It is shown that in several frequency bands radiation is emitted. These different frequencies are identified as belonging to the FEL instability and another mechanism, probably the gyrotron instability. The measurements show apparently a competition between these mechanisms. The experimentally determined growth rate is somewhat larger than the theoretical one, indicating that the FEL does not operate as a true amplifier.
\end{abstract}

\section{Introduction}

A standard diagnostic for an experimental study and comparison with theory is the measurement of the spatial evolution of the emitted power by changing the interaction length (e.g. by stopping the electrons, as described below) $[1,2]$. More information can be obtained by measuring the evolution of the spectrum as well, which is the subject of this paper. The settings chosen for the magnetic fields (see below) correspond to an average steady state perpendicular velocity $\beta_{1}=0.17$ at a total beam energy $\gamma$ of 1.974 . It is found that this point is not far away from magneto-resonance. Earlier experiments [3] have indicated that in such a case the FEL as well as the gyrotron interaction is possible. In this region, proper injection of the electron beam becomes very important because oscillations around the steady state axial velocity are easily excited.

\section{Experimental set-up}

The general set-up of the Twente Raman FEL has already been thoroughly described elsewhere [4]. The laser uses a pulsed bifilar undulator consisting of 40 periods of $3 \mathrm{~cm}$ each, with a tapering of 6 periods at the entrance. A Marx generator produces voltage pulses of $100 \mathrm{~ns}$ duration with a nominal value of $500 \mathrm{kV}$. The current is approximately $190 \mathrm{~A}$.

\footnotetext{
* Corresponding author. Tel. + 3153833969, fax +3153338 065, c-mail: paolo(age.tn.utwente.nl.
}

In order to study the spectral evolution, the interaction length was varied by stopping the electron beam along the undulator using a kicker magnet. Calculations show that a kicker magnetic field of $0.3 \mathrm{~T}$ is sufficient to stop the electron beam within $2 \mathrm{~cm}$. This has been confirmed by experiments. The diagnostic of the produced radiation consists of a Joule meter and a set of high pass filters covering the $\mathrm{K}_{\mathrm{a}}$-band [4]. The filters produce equidistant wavelength bands with a width of $1 \mathrm{~mm}$. The two detection methods cannot be used simultaneously. All measurements reported here are obtained for an axial guide field of $B_{z}=1.02 \mathrm{~T}$ and an undulator field $B_{\mathrm{u}}=0.19 \mathrm{~T}$. Linear theory [5] predicts the FEL interaction with the $\mathrm{TE}_{11}$ to occur at $30 \mathrm{GHz}(10 \mathrm{~mm})$ for these settings.

The Marx generator has an inherent spread between successive shots. In analyzing the results only shots were selected with a maximum spread of $2 \%$ in the accelerating voltage during the pulse. The field emission diode produces an inherent increase in the current during a shot. This was limited to a maximum increase of $10 \%$. Shot to shot fluctuations were $2 \%$ and $10 \%$ for voltage and current respectively.

\section{Spatial evolution of the spectrum}

In the first experiments the total emitted energy was measured using the Joule meter and a high-pass filter with a cut-off frequency of $21 \mathrm{GHz}$. In the case of the cut-off filter the time dependent signal was integrated to obtain the total received energy. The results are shown in Fig. 1 where the energy is plotted versus the interaction length of the electron beam. Below an interaction length of $60 \mathrm{~cm}$ 


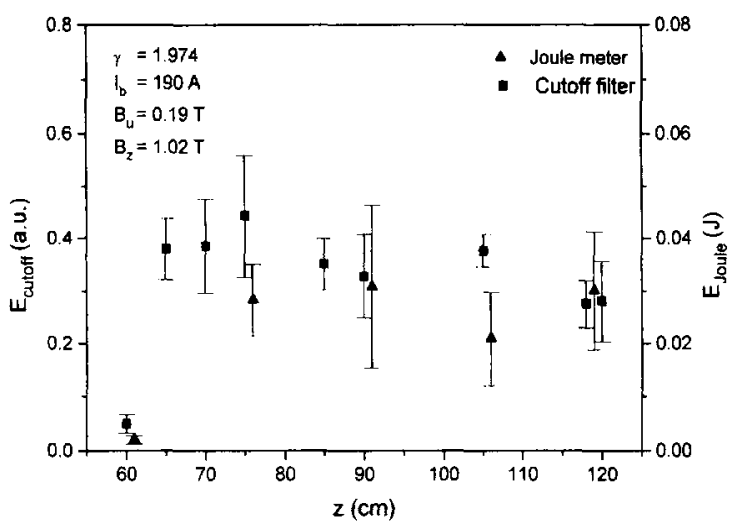

Fig. 1. Total energy measured with the Joule meter (triangles) and with a high pass filter with a cutoff frequency of $21 \mathrm{GHz}$ (squares) as a function of the interaction length.

no signal was found above the background noise. The general behaviour of the two types of measurement is quite the same apart for an increase in output energy observed at the end of the undulator by the Joule meter and not by the cut-off filter. This indicates that the radiation shifts to longer wavelengths at the end of the undulator. It is clear from rig. 1 that a large growth appears up to about $65 \mathrm{~cm}$ and the laser output seems to be more or less constant for larger interaction lengths. Assuming exponential growth between 60 and $65 \mathrm{~cm}$ a "power" growth rate of $\sim 0.41$ $\mathrm{cm}^{-1}$ is obtained for the total radiation field.

The spectral evolution was measured in the same way as the total energy, however here only cut-off filters were used. The difference in energy as measured by two filters with successive cut-off frequencies was attributed to radiation emitted in the frequency band between these cut-off frequencies. The first observation which can be made from these measurements is that all frequency bands show a local maximum for an interaction length between 65 and $80 \mathrm{~cm}$. Second the different frequencies show more than one local maximum at different values for the interaction length. Some examples are given in Fig. 2. A typical example of the complete spectrum (also indicating a typical spread in the measurements) is shown in Fig. 3. Note that the measurements in Fig. 2 (represented by symbols) are connected by straight lines for convenience only. For example the $33.3 \mathrm{GHz}$ line grows from approximately zero to a maximum when the length increases from 60 to $65 \mathrm{~cm}$ and drops back to zero for larger lengths. Only at the end of the undulator $(120 \mathrm{~cm})$ some energy is again emitted at this frequency. It is further observed that the growth in some frequency bands is complementary to that in other frequency bands. It is found that at the $25 \mathrm{GHz}$ band the energy increases when it decreases for the $30 \mathrm{GHz}$ and 23 $\mathrm{GHz}$ bands (as is shown in Fig. 2). It seems that some kind of competition between the different frequencies is present.

Assuming that for an interaction length less than $60 \mathrm{~cm}$ the radiation is growing from noise and is in the exponen-

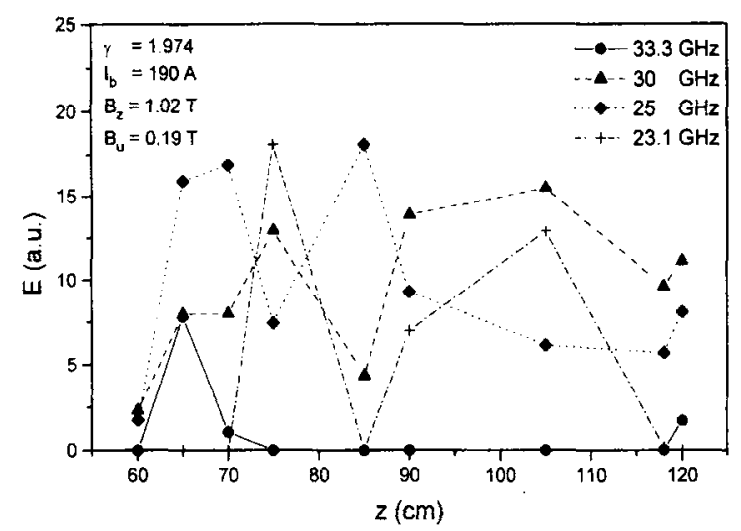

Fig. 2. The energy $E$ emitted in different frequency bands as a function of the interaction length. The center frequency is indicated. All bands have a spectral width of $1 \mathrm{~mm}$ (i.e. they vary in the frequency domain).

tial growth regime up to the first local maximum, the growth rate can be derived from it. Fig. 2 already indicates that the different frequency bands have different growth rates. At $65 \mathrm{~cm}$ the largest contribution to the total energy comes from the $25 \mathrm{GHz}$ band. This band shows a "power" growth rate of $\sim 0.44 \mathrm{~cm}^{-1}$, which is somewhat larger than the one found for the total energy. The $30 \mathrm{GHz}$ band has at this position a "power" growth rate of $\sim 0.24$ $\mathrm{cm}^{-1}$. For other frequency bands the growth rates cannot be determined as the signal could not be distinguished from noise in the detectors at $60 \mathrm{~cm}$ interaction length.

The undulator did not contain a taper at the exit. As a rcsult the magnetic ficld will show a spike at the exit [6]. The question arises whether this spike can excite electron orbits required for gyrotron type interaction in the region after the undulator. To study this experimentally the electron beam was stopped just before the end of the undulator using the kicker magnet. In another series of measurements

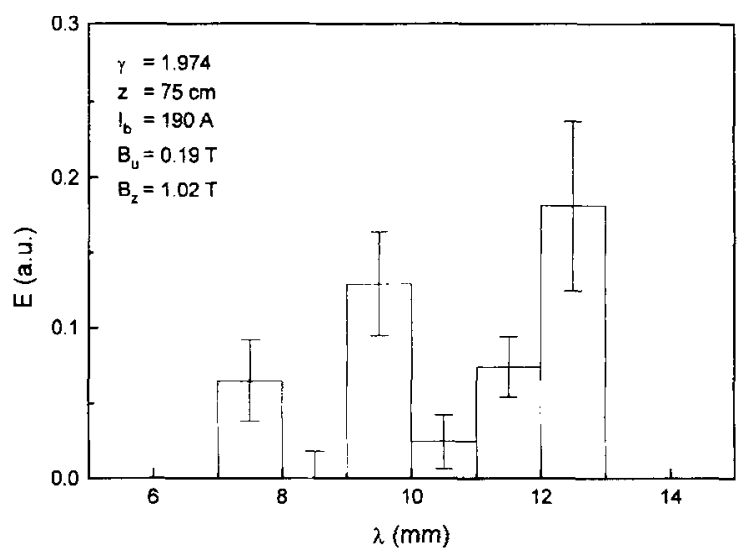

Fig. 3. Typical example of spectrum measured at an interaction length of $75 \mathrm{~cm}$. The error bars indicate the shot to shot spread in the measured energy. 
it was allowed to propagate through the undulator into the region with only the axial guide field. No significant differences were found in the output energy as well as in the measured spectra. The spike will most likely disturb the electron beam at the exit of the undulator but the beam seems not to couple to the radiation field any more. The guide field starts to diverge just behind the exit of the undulator. This will also make it difficult to couple the electron beam to the radiation field through a gyrotron-like interaction.

\section{Preliminary comparison with non-linear theory}

To compare the measured results with theory a non-linear model describing an amplifier set-up was developed. This model is basically the one described in Ref. [7]. However, it was modified to include the complete three dimensional electron orbits. At the moment the code only allows interaction of the electron beam to take place with the $\mathrm{TE}_{11}$ mode. Higher order modes will be included in the future.

Calculations were performed for the experimental parameters given above. Only interaction was found around $30 \mathrm{GHz}$. No interaction with the $\mathrm{TE}_{11}$ was found at other frequencies within the $\mathbf{K}_{\mathrm{a}}$-band. The saturated power level was about $12 \mathrm{MW}$. The exponential (power) growth rate found was $0.182 \mathrm{~cm}^{-1}$. The growth rate derived from the measured spectral evolution is somewhat larger. This could well be explained by a small reflection from the outcoupling window used in these experiments $[3,4]$. From the Joule meter measurements the maximum total energy emitted was about $30 \mathrm{~mJ}$. Using the pulse duration of the electron beam ( $100 \mathrm{~ns}$ ) an average power of about 0.03 MW is found. The time structure of the radiation pulse shows many spikes so that the peak power within the radiation pulse can be an order of magnitude higher. This is still far below the levels predicted by the numerical code. Two explanations can be given. First we have observed growth at frequencies different from $30 \mathrm{GHz}$ which is not predicted by the numerical code. Two possibilities arise. Interaction takes place with higher order modes or a second type of interaction not described by the non-linear model takes place. The latter could be gyrotron interaction which has been found before [3]. For the experimental parameters this interaction could very well be initiated by the injection of the electron beam in the undulator [4]. A second explanation could be found in the quality of the electron beam. To reduce the increase of the current with time, which results from the expanding plasma in the field emission diode, the anode-cathode distance was increased. This may have influenced the quality of the electron beam more than was cxpected. A reduced quality will affect the growth rate and maximum power which can be obtained. It does not explain, however, the growth observed at other frequencies. Comparison between the experimental and theoretical growth rate seems not to indicate a serious beam degradation. However this needs to be checked. Mos! likely the measurements are a result of a combination of the two effects. Furthermore it is not clear how the apparent competition which we observe between growth in different frequency bands will influence the saturation levels.

\section{Discussion}

For the parameters investigated a complex evolution of the spectrum was found. This evolution seems to indicate a competition between radiation emitted in several frequency bands, one of which is identified as corresponding to the FEL interaction. As the operating point was rather close to magneto-resonance the complicated electron orbits could very well couple to the FEL as well as the gyrotron instability. The growth rate found experimentally was somewhat larger than the theoretical one, which indicates that some of the radiation is reflected at the outcoupling window. The system is therefore not a true amplifier. The frequencies which could not be identified as FEL interaction are generated inside the undulator as no significant difference was found when the beam was allowed to propagate to the end of the axial guide field and when it was stopped just before the exit of the undulator.

\section{References}

[1] J. Fajans, J.S. Wurtcle. G. Bekefi, D.S. Knowles and K. Xu, Phys. Rev. Lett. 57 (1986) 579.

[2] A. Bhattacharjee, S.Y. Cai, S.P. Chang, J.W. Dodd, A. Fruchtman and T.C. Marshall, Phys. Rev. A 40 (1989) 5081.

[3] P.J.M. van der Slot and W.J. Witteman, Nucl. Instr. and Meth A 331 (1993) 140.

[4] P.J.M. van der Slot, Ph.D. Thesis, University of Twente, The Netherlands (1992).

[5] J. Fajans, G. Bekcfi. Y.Z. Yin and B. Lax, Phys. Fluids 28 (1985) 1995.

[6] J. Fajans, J. Appl. Phys. 55 (1984) 43.

[7] J.S. Wurtele, R. Chu and J. Fajans, Phys. Fluids B 2 (1990) 1626. 